\title{
EL PROBLEMA DE LA CUEVA. ELEMENTOS PARA UN ANÁLISIS DIDÁCTICO DE LOS PROBLEMAS DE PROBABILIDAD
}

\author{
HUERTA PALAU, M. PEDRO \\ Departament de Didàctica de la Matemàtica \\ Escola de Magisteri. Universitat de València. 46071 València
}

\begin{abstract}
Resumen. En este artículo pretendemos mostrar qué tipos de análisis pueden hacerse de los problemas de probabilidad que sean útiles para la formación de profesores de matemáticas. Usamos como herramientas de análisis elementos de un modelo de competencia en la resolución de problemas que pueden ser de utilidad para los problemas de probabilidad: herramientas heurísticas, destrezas heurísticas y métodos de resolución con contenido heurístico, al cual incorporamos la manera cómo se realizan los análisis; eso es, desde la introspección y desde la posición del resolutor ideal, la noción de sistema matemático de signos y la consideración de diferentes estratos de este sistema matemático de signos que permiten resolver el problema objeto de análisis. Palabras clave. Probabilidad, formación de profesores de matemáticas, resolución de problemas, heurística.

Summary. In this report we intend to show what kinds of analysis can be made of problems of probability so as to make them useful for the training of mathematics teachers. As tools for the analysis we use elements of a pattern of competence in problem solving useful for problems of probability: heuristic tools, heuristic skills and solving methods with an heuristic content, to which we add the way how we make the analyses, which is, from the introspection and the position of the ideal solver, the notion of mathematical system of signs and the consideration of different strata of this mathematical system of signs that allow to solve the problems being analysed.

Keywords. Probability, preservice mathematics teaching, problem solving, heuristics.
\end{abstract}

\section{INTRODUCCIÓN}

El problema que se presenta en este trabajo es uno de aquellos problemas que sirvieron en su momento para experimentar la transición de un sistema educativo a otro. Sirvió también en cursos de perfeccionamiento de profesores de matemáticas de secundaria que estaban en transición de un sistema educativo a otro. Pero la verdad es que pocos profesores tuvieron -tuvimos-esta oportunidad, la de experimentar, la de actualizarse, la de plantearse y resolver problemas de la forma en la que lo hacemos aquí. Hoy, probablemente, pocos estudiantes de secundaria tengan la oportunidad de resolver el problema al que nos vamos a referir, y esto, seguramente, por dos motivos fundamentales: uno, porque la transición ya se hizo - con el resultado que todos conocemosy, por tanto, los profesores de hoy no están en la misma situación de entonces, dando como consecuencia que hay poco tiempo para actualizase y experimentar; y, dos, no es un problema habitual en los libros de texto de matemáticas de secundaria, al menos de las editoriales más conocidas, aunque sí puede encontrarse una propuesta parecida en un proyecto curricular de las matemáticas para la secundaria obligatoria (Grupo Cero, 1995). Hoy, el enunciado del problema, al que hago referencia más adelante, puede encontrarse en los materiales de trabajo para las matemáticas de la enseñanza secundaria obligatoria que la Conselleria d'Educació i Ciència de la Generalitat Valenciana editó a principio de los noventa como resultado de aquella experimentación. Recordemos que la ley que reguló estas enseñanzas es del año 1992, por lo que en aquellas fechas el profesorado de matemáticas que experimentaba y anticipaba la reforma de la enseñanza secundaria contaba casi exclusivamente 
con estos materiales para sus clases, incluyendo éstos pequeñas orientaciones metodológicas basadas en el resultado de su experimentación en épocas pasadas.

No es sorprendente que los impedimentos para una enseñanza efectiva de la probabilidad y la estadística en las escuelas sean los mismos que los que tiene la resolución de problemas. La fuerte conexión entre esos dos dominios de las matemáticas ya han sido señaladas con anterioridad (Shaughnessy, 1992). Por ejemplo, la enseñanza y el aprendizaje de la probabilidad y la estadística implican construir modelos de fenómenos físicos, desarrollar y usar estrategias (tales como la simulación o el conteo) y comparación y evaluación de diferentes enfoques a los problemas para controlar posibles comprensiones y representaciones equivocadas. En este sentido, enseñar probabilidad y estadística es enseñar resolución de problemas, aunque en un dominio particular. Además, los conocimientos de los profesores tanto en probabilidad y estadística como en resolución de problemas son, en general, débiles y, en algunos casos, inexistentes. Esto no es fallo de ellos, cuando históricamente los programas de formación de profesores no han incluido sistemáticamente ni la probabilidad y estadística ni la resolución de problemas para profesores de matemáticas en activo. Finalmente, la resolución de problemas y la probabilidad y estadística son ambos, en algún sentido, recién llegados al bloque de matemáticas, al menos desde la perspectiva de su inclusión en el currículo de secundaria y especialmente si uno lo compara con la tradición de otras partes del currículo de matemáticas (Shaughnessy, 1992).

Los análisis que se mostrarán en este artículo deben situarse en el contexto particular de la formación de profesores de matemáticas ${ }^{1}$. No en balde, con énfasis diferentes, los hemos usado en nuestras clases con estudiantes de magisterio, con estudiantes de la facultad de matemáticas y con estudiantes de tercer ciclo en didáctica de las matemáticas. Pretenden analizar el proceso de resolución del problema, que hemos bautizado como el problema de la cueva, desde algunos de los elementos del modelo de competencia de la pura resolución de problemas (Puig, 1996) en lo que puede ser pertinente para este problema de probabilidad.

\section{LOS ELEMENTOS PARA UN ANÁLISIS DI- DACTICO}

En el contexto particular en el que se presenta este trabajo, los análisis que son pertinentes hacer del problema de la cueva tienen en cuenta elementos que, siendo de orígenes diferentes, confluyen aquí en lo que hemos titulado simplemente como el problema de la cueva. Estos elementos son:

a) el mundo de la resolución de problemas que contempla éstos en relación con los sistemas matemáticos de signos (Filloy, 1999; Puig, 1997) con el que se resuelven (Puig, 1996); b) el escenario en que se desarrolla, curso de didáctica de la probabilidad y la estadística para la formación de profesores (Huerta, 2000);

c) el nivel de análisis con el que se estudia el proceso de resolución y que en este caso considera el nivel macroscópico junto con la introspección -tomando en consideración la figura del resolutor ideal (Puig y Cerdán, 1988);

d) los elementos de un modelo de competencia tomados de la pura resolución de problemas y que tienen vocación de convertirse en un modelo de competencia en la resolución de problemas de probabilidad -modelo que está por construirse o construyéndose: herramientas heurísticas $(\mathrm{HH})$, destrezas heurísticas (DH) y métodos de resolución con contenido heurístico (MH) (Puig, 1996);

e) las DH para la resolución de problemas de probabilidad (Fischbein, 1977; Engel, 1975a, 1975b);

f) los procesos de resolución y los conceptos matemáticos implicados (Huerta, 2000).

Las referencias que se incluyen en la lista de elementos que se consideran para el análisis didáctico del problema de la cueva dan cuenta abundantemente de ellos. Nuestro propósito aquí es ponerlos en juego durante la resolución del problema, de manera que permita realizar los análisis pertinentes y que se incluyen a lo largo del texto. Así, la resolución del problema se presenta y se organiza atendiendo al primero de los elementos citados, los sistemas matemáticos de signos. El escenario $\mathrm{y}$, por tanto, el sentido con el que se deben considerar los análisis, forma parte de un escenario más amplio: un curso de didáctica de la probabilidad y la estadística para la formación de profesores. Por otra parte, quien resuelve el problema es un resolutor ideal que sabe las cosas que hay que hacer en cada momento y por qué hay que hacerlas, tomando en consideración las $\mathrm{HH}, \mathrm{DH}$ y $\mathrm{MH}$ del mundo de la pura resolución de problemas que son pertinentes para el problema de probabilidad que se está analizando. Finalmente se consideran los sistemas de representación, considerados aquí como DH, que permiten visualizar los conceptos matemáticos implicados en el proceso de resolución del problema y las reglas de cálculo asociadas, como teoremas en acto, que permiten resolverlo.

\section{EL PROBLEMA DE LA CUEVA. LOS ENUN- CIADOS}

\section{El problema de la cueva para los estudiantes de primaria y secundaria}

Veintisiete exploradores están perdidos en una cueva de la que parten tres caminos. Uno de ellos conduce al exterior en una hora. Los otros dos no tienen salida: si entran por uno de ellos, vuelven a la cueva en dos días, y si entran por el otro, en tres días. 
Como no llevan ninguna luz y la cueva está obscura y llena de obstáculos, eligen, cada vez que hacen un intento por salir, uno de los tres caminos al azar.

Si cada explorador sólo tiene comida para sobrevivir durante menos de seis días, ¿cuántos de los 27 exploradores crees que lograrán salir de la cueva?

\section{El problema del estudiante para profesor ${ }^{2}$}

\section{El enunciado}

Veintisiete exploradores están perdidos en una cueva de la que parten tres caminos. Uno de ellos conduce al exterior en una hora. Los otros dos no tienen salida: si entran por uno de ellos, vuelven a la cueva en dos días; y si entran por el otro, en tres días.

Como no llevan ninguna luz y la cueva está obscura y llena de obstáculos, eligen, cada vez que hacen un intento por salir, uno de los tres caminos al azar.

Supongamos que:

a) Cada explorador sólo tiene comida para sobrevivir durante menos de seis días.

b) La comida no es problema, siempre hay.

Las tareas. El estudiante como resolutor del problema

1) Para cada uno de los supuestos anteriores, hallar la probabilidad que tiene un explorador de salir de la cueva.

2) Para cada uno de los supuestos anteriores, hallar el número esperado de exploradores que saldrán de la cueva y el tiempo esperado para que finalice el proceso.

3) Para cada uno de los supuestos anteriores, diseñar una simulación del problema de manera que permita resolver el problema por simulación. Prestar atención a los siguientes aspectos:

a) Generadores de azar posibles.

b) ¿Cómo deben usarse los generadores de azar y por qué?

c) ¿Cómo han de organizarse los datos con el fin de facilitar una respuesta a las preguntas del problema?

d) ¿Qué significados pueden construirse de los conceptos de probabilidad y esperanza matemática, de los apartados 1 y 2 , derivados de su uso en la resolución del problema por simulación?

e) La clase de secundaria de la que sois profesores está compuesta por 30 estudiantes. La probabilidad que asigne por simulación cada estudiante o la clase queremos que no exceda en más de un $\varepsilon$ de la probabilidad teórica -calculada por nosotros con anterioridad-con un grado de confianza $\uparrow$. ¿Cuántas simulaciones deberá hacer como mínimo cada estudiante?

\section{La propuesta de trabajo del estudiante para profe- sor. El estudiante para profesor como observador de los procesos de resolución}

En los primeros apartados se propone a los estudiantes para profesor que el problema se resuelva en más de una forma, de manera que cada solución del problema implique poseer más de un componente de análisis:

a) el componente formal, que implica el uso del sistema matemático de signos más abstracto;

b) la aproximación a la secundaria, que implica el uso de un sistema matemático de signos menos abstracto: sistemas de representación y de reglas de cálculo asociadas;

c) la aproximación a la primaria con el uso del sistema matemático de signos menos abstracto implicado en el juego y el ábaco probabilístico (Engel, 1975b).

En el apartado siguiente se sugiere que el problema se resuelva también por simulación y a partir de la información y, por tanto, de los datos, que ésta proporciona. Se ha de analizar la simulación desde una doble vertiente, como método de resolución de problemas de probabilidad y como contexto particular de enseñanza en el que los estudiantes de primaria o secundaria adquieran experiencias sobre las naturalezas diversas de los conceptos de probabilidad y de esperanza matemática.

\section{LOS PROCESOS DE RESOLUCIÓN DEL PROBLEMA DE LA CUEVA ANALIZADOS DESDE EL SISTEMA MATEMÁTICO DE SIGNOS QUE LO RESUELVE}

Hemos indicado que uno de los mundos posibles en el que ubicar la resolución de problemas es aquél en el que los problemas se miran desde los sistemas matemáticos de signos que los resuelve. Procede, pues, tomar una posición respecto de esta noción. Así, adoptaremos la posición que describe Puig (1997) refiriendo que, en la actividad matemática, «la combinación de todos los signos que se usan -ya sean signos matemáticos o un sistema de signos matemáticos, el lenguaje vernáculo y otros medios de representación, quizás- constituyen un sistema matemático de signos (SMS)» (p. 68) y lo que interesa no es el estudio de los signos en sí mismos, sino que «desde el punto de vista de la didáctica es más interesante los procesos de significación y de producción de sentido que el estudio de los signos y sus tipos» (p. 68). En consecuencia, lo que mostraremos será el sistema de signos que permite resolver el problema de la cueva, formado por diferentes estratos que determinan niveles de abstracción crecientes, tanto en el uso de los signos como en la significación que el uso de esos signos dota a los conceptos de probabilidad implicados en la resolución del problema. 


\section{El estrato más abstracto del SMS}

Si indicamos por $E_{\mathrm{i}}$, para $i=1,2,3$, el espacio de pruebas del intento $i$, el espacio de pruebas del problema es $X=E_{1} x E_{2} x E_{3}$. En este espacio, una posibilidad-suceso elemental- de salir de la cueva la podemos representar por un vector de tres componentes no todos nulos ${ }^{3}$ $(i, j, k)$, de manera que el conjunto de todas las posibilidades nos permite construir el espacio muestral asociado al proceso aleatorio implicado en nuestro problema. Los componentes $i, j, k$ de este vector indicarán la posibilidad del explorador en cada intento, de manera que los valores posibles 1, 2 o 3 indicarán escoger la posibilidad de la puerta que lleva al exterior en 1 hora, al interior después de 2 días o al interior después de 3 días, respectivamente. Así, para cada explorador:

$X^{1}=\{(i, j, k) /$ si $i=1$, entonces $j=k=0$; si $i \neq 1$ y $j=1$, entonces $k=0$ pero si $j \neq 1$, entonces $k=1\}$, que junto con $X^{0}=\{(i, j, k) / i, j$ y $k$ distintos de 1 ; o bien si $i=2,3, \mathrm{y}$ $j=2,3$, entonces $k=2,3$, y si $i=j=3$ entonces $k=0\}$ permiten definir el espacio muestral $X$ como unión disjunta de $X^{1}$ y $X^{0}, X=X^{1} \cup X^{0}$.

Definimos la función de probabilidad $p$ sobre $X$ por $p(x)$ $=(1 / 3)^{n}$, donde $n$ es el número máximo de componentes no nulos del vector $(i, j, k)$ que define $a x \in X$. Definimos también el suceso salir de la cueva por el conjunto $X^{1} \mathrm{y}$ el suceso no salir de la cueva por el conjunto $X^{0}$, que por construcción son conjuntos disjuntos y, a su vez, unión disjunta de los vectores $(i, j, k)$ que los caracteriza. De esta manera, las probabilidades de los sucesos considerados están definidas por:

$p\left(X^{1}\right)=\sum n(1 / 3)^{\mathbf{n}}$, siendo $n=1,2,3$ y por $p\left(X^{0}\right)=1-p\left(X^{1}\right)$.

Sea $Z$ la variable aleatoria que asigna a cada $x \in E X^{1}$ el número de exploradores que salen de la cueva y que para cada $\mathrm{x} \in \mathrm{EX}^{0}$ el número de exploradores que no salen de la cueva. $Z$ se distribuye según una binomial de parámetros $(27,2 / 3)$, siendo $p=2 / 3$ la probabilidad de salir de la cueva, lo que permite resolver la pregunta del problema $E(Z)=27 \times 2 / 3=18$ exploradores.

El uso del estrato más abstracto del SMS que nos permite resolver el problema exige, consecuentemente, que el proceso de resolución tenga un desarrollo lógico con la determinación del espacio de pruebas, de las posibilidades o sucesos elementales, del espacio muestral, de la función «probabilidad» sobre el espacio muestral, de los sucesos implicados, de la variable aleatoria y de la distribución de probabilidad que organiza esta variable aleatoria.

\section{El estrato intermedio del SMS}

Vamos a considerar como estrato intermedio del SMS aquél que usa como signos los propios de un sistema de representación, que en nuestro caso es un árbol y los caminos que constituyen el árbol, y las reglas de cálculo asociadas al sistema de representación.
El espacio de pruebas consiste en un árbol de longitud ${ }^{4}$ 3 que proporciona 27 caminos posibles. Una posibilidad es, pues, cualquier camino de longitud 3, como éstos:

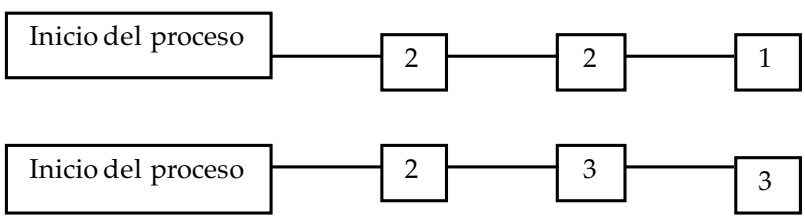

Un observador externo ${ }^{5}$ del proceso puede observar cuál es el final del mismo -que depende de las distintas opciones que puede escoger un explorador-, dejando ramas del árbol vivas o cerrándolas de manera que los caminos posibles de salir de la cueva se extiendan desde la longitud 1 hasta la longitud 3, como éstos:

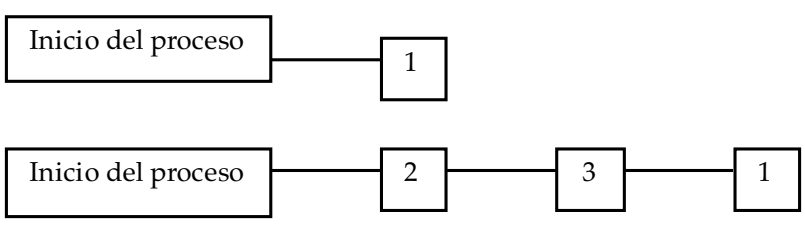

que representan dos posibilidades de salir de la cueva, o éstos:

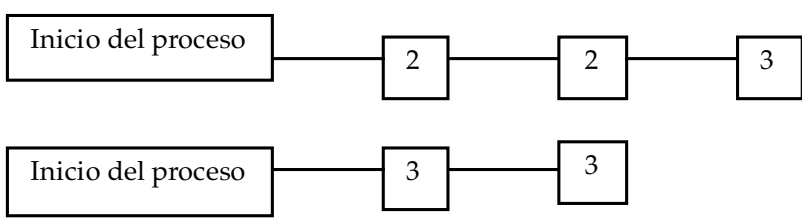

que representan dos posibilidades que no permiten salir de la cueva.

De otra manera, un explorador sale de la cueva si la posibilidad escogida, el camino del árbol, acaba en 1. En caso contrario, no sale de la cueva. Así, el espacio muestral para el observador externo está formado por 13 caminos de longitudes variables, de los cuales 6 conducen al exterior de la cueva (suceso $X^{I}$ ) y los 7 restantes no $\left(X^{0}\right)$. Los sucesos $X^{1}$ y $X^{0}$ pueden verse como subconjuntos del espacio muestral -el árbol-o no, pero en todo caso los sucesos son representaciones de trozos del espacio muestral.

La construcción del espacio muestral mediante un árbol lleva asociada la asignación de probabilidades de cada una de las posibilidades de escoger una puerta por parte de los exploradores. La equiprobabilidad que subyace en cualquiera de las elecciones permite asignar la probabilidad $1 / 3$ a cada uno de los tramos del camino, como puede verse en el ejemplo siguiente: 


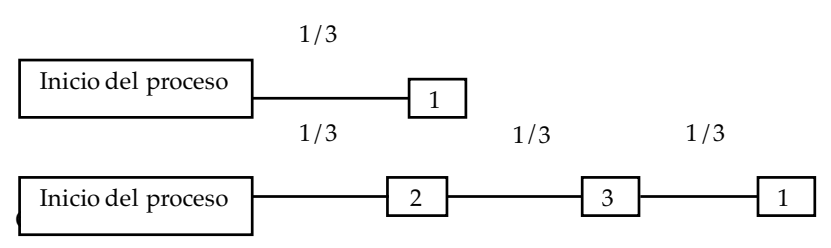

Una interpretación del significado de la probabilidad recién asignada, cuando ahora intervienen los 27 exploradores, permite determinar el número esperado de exploradores que se espera que recorran cada uno de los caminos. Así, con los ejemplos anteriores, podemos determinar cuántos exploradores se espera que transiten por esos caminos:

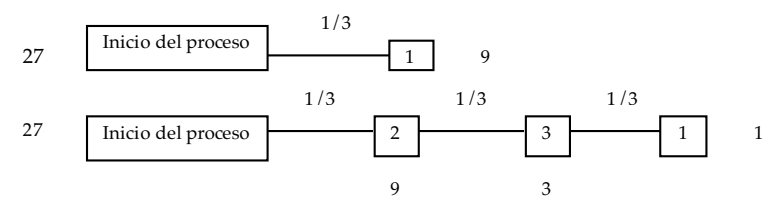

Un recuento de los números que hay al final de los caminos nos permite saber el número de exploradores que se espera que salgan de la cueva, lo cual constituye la respuesta al problema planteado.

El proceso de resolución del problema que hemos desarrollado con el uso del estrato intermedio del SMS nos ha permitido responder al problema sin necesidad de recurrir al desarrollo lógico que exige el uso del estrato más abstracto del SMS. Otras cuestiones sobre la misma situación problemática, que ahora son colaterales a la pregunta original del problema, pueden plantearse con el fin de aprovechar el sistema de representación que describe el espacio de posibilidades asociado a esa situación problemática y su potencial heurístico (Fishbein, 1977) como herramienta que permite resolver otros problemas relacionados. Una de estas cuestiones es la probabilidad de salir de la cueva.

Resolver esta última cuestión exige introducir reglas de cálculo asociadas a caminos y árboles (Engel, 1975a). Así, la regla del producto para caminos nos permite obtener una función de probabilidad definida sobre el árbol:

Para cualquier camino, y dada la equiprobabilidad encontrada a lo largo de él, $p$ (camino $)=(1 / 3)^{n}$, siendo $n$ la longitud del camino.

Así, por ejemplo:

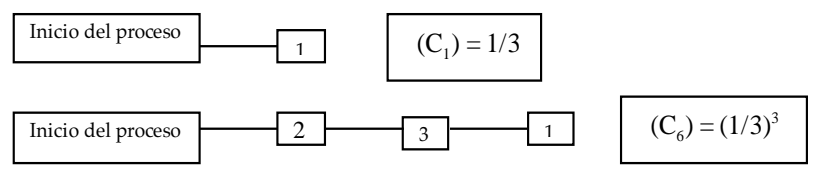

Teniendo en cuenta todas las probabilidades de los caminos $C i$, con $i=1,2 \ldots, 13$, en los términos anteriores y descrito el suceso salir de la cueva por los caminos 1 , 2, 3, 6, 9 y 10 , calcular su probabilidad exige una nueva regla, la regla de la suma (Engel, 1975a), que nos permite obtener la probabilidad de un suceso que puede producirse por una variedad de caminos posibles.

La vuelta a la pregunta original, ahora con la información adicional de la probabilidad con la que puede salirse de la cueva, permite explorar el número esperado de exploradores que se espera que salgan de la cueva desde la perspectiva de una nueva regla de cálculo, la regla del valor medio para caminos y árboles (Engel, 1975b), en el que la ponderación que se realiza en cada uno de los tramos de un camino exige el uso de la probabilidad con la que se transita por ese tramo, lo cual pondera el número de exploradores que se espera que transiten por él. La variable aleatoria «número de exploradores» que se espera que salgan de la cueva se interpreta como la suma de un número de valores esperados de cada una de las variables aleatorias que define cada uno de los caminos por los que se puede salir al exterior.

\section{El estrato menos abstracto del SMS}

Este estrato es considerado así porque en él los signos con los que se va a resolver el problema están representados por fichas de juego, como las del parchís. Se considera además un tablero de juego, la representación de la cueva del problema y las reglas para jugar, esto es, la reglas para mover fichas y para finalizar el juego.

El espacio de pruebas en este estrato del SMS lo constituye el tablero de juego con 27 fichas blancas en su interior. El juego, o las reglas del juego, exige que el jugador tenga en cuenta las restricciones de cada una de las fichas para los diferentes intentos por salir de la cueva. Una forma de tener esto en cuenta es introducir fichas con colores diferentes cuyo significado sea, para un observador externo, la situación del explorador en el proceso aleatorio. Así, por ejemplo, una ficha verde significaría un explorador en el exterior de la cueva después de haber escogido la puerta conducente al exterior en una hora. Una ficha roja en mitad de un camino significaría que un explorador ha agotado sus recursos y se encuentra en el interior de la cueva. De esta forma, el espacio de posibilidades estaría formado por combinaciones de fichas de colores que el observador externo, el estudiante, observaría o bien dentro de la cueva o bien fuera de la cueva.

Las reglas del juego están relacionadas con la interpretación intuitiva de la equiprobabilidad de las tres opciones del explorador cada vez que hace un intento o, de un modo mucho más general, con la interpretación de la probabilidad por la frecuencia relativa. En nuestro caso, mover fichas consiste en repartir equitativamente los 27 exploradores por las tres opciones de la cueva, simular la historia del problema (salir fuera, volver a la cueva) y repetir hasta que el proceso quede terminado. 
El suceso salir de la cueva estaría representado por el conjunto de fichas fuera de la cueva, mientras que el suceso no salir de la cueva lo estaría por el conjunto de fichas que han permanecido en el interior de la cueva. Las fichas, en consecuencia, tienen un doble significado en función de los colores que se hayan asignado. Así, un significado asociado al suceso es el que se deriva de la posición de cada ficha en el tablero de juego, dentro o fuera de la cueva; el otro significado está asociado al color de cada ficha fuera de la cueva y que indica la manera en la que se ha producido el suceso salir fuera, a la primera ( 1 hora), a la segunda (2 o 3 días y una hora), o a la tercera ( 4 días y 1 hora o 5 días y 1 hora). El color de las fichas dentro de la cueva no son relevantes para las cuestiones del problema.

El estrato del SMS que estamos considerando ahora está formado por tres grupos de fichas que representan el espacio de posibilidades $(X)$ y los sucesos salir de la cueva $\left(X^{I}\right)$ y no salir de la cueva $\left(X^{0}\right)$. Claramente card $(X)=\operatorname{card}\left(X^{I}\right)+\operatorname{card}\left(X^{0}\right)$.

El problema está resuelto con sólo determinar $X^{1}$ y su cardinal. Pero, igual que con el estrato intermedio, pueden resolverse otras cuestiones relacionadas. Así, la probabilidad del suceso $X^{1}$, que compara los tamaños de los conjuntos fichas representados por $X^{1}$ y $X$, y que puede expresarse como «tantos de tantos», puede definirse o bien como la relación -en términos de razónentre el número de fichas del conjunto $X^{l}$ y el número de fichas del conjunto $X$ o bien, perdiendo la cualidad del color de la ficha, como la relación entre el número de fichas que salen de la cueva y el número de fichas que entraron. Análogamente, puede determinarse la probabilidad del suceso representado por $X^{0}$.

\section{LA EXTENSIÓN DEL PROBLEMA. NUEVO ESPACIO DE PROBLEMAS. NUEVO SMS Y NUEVOS ESTRATOS DE ESTE SMS}

La restricción «tienen alimentos para menos de seis días» convierte el proceso aleatorio en finito. Eliminar esta restricción lo convierte en un proceso infinito. El nuevo problema: «Veintisiete exploradores están perdidos en una cueva de la que parten tres caminos. Uno de ellos conduce al exterior en una hora. Los otros dos no tienen salida: si entran por uno de ellos, vuelven a la cueva en dos días, y si entran por el otro, en tres días. Como no llevan ninguna luz y la cueva está obscura y llena de obstáculos, eligen, cada vez que hacen un intento por salir, uno de los tres caminos al azar. ¿Cuántos de los 27 exploradores crees que lograrán salir de la cueva?» introduce a los estudiantes en un nuevo espacio de problemas que se conocen como cadenas de Markov (Gordon, 1997).

\section{El estrato más abstracto del nuevo SMS}

Para cada explorador, el espacio de posibilidades puede representarse por:
$\mathrm{X}=\{(i, j \ldots k, 0,0 \ldots 0 \ldots)$, con $i, j, k 1,2,3$, siendo $k=1$ si $h \neq 1\}$, donde, por ejemplo, $(2,3,1,0 \ldots 0 \ldots)$ o $(2,3,3,2,3,2,1,0 \ldots 0 \ldots)$ representan la posibilidad de salir de la cueva al tercer intento o al sexto intento, respectivamente.

Un observador, considerando el proceso aleatorio como un sistema, observa dos estados posibles:

$E_{l}$, el sistema está en el estado 1 (dentro de la cueva) $E_{2}$, el sistema está en el estado 2 (fuera de la cueva).

Y la siguiente relación entre probabilidades condicionales:

$p$ ( $E_{2} /$ estamos en $E_{1}$ después de $\mathrm{n}$ intentos anteriores) $=p\left(E_{2} /\right.$ estamos en $E_{l}$ en el intento $\left.n-1\right)$, es decir, la propiedad de Markov.

Podemos preguntarnos por la probabilidad de alcanzar un determinado estado «tarde o temprano» y la duración media del proceso aleatorio. Para ello, hemos de considerar dos tipos de probabilidades:

$p_{i j}=$ probabilidad de transitar en un solo paso desde el estado $E_{i}$ al estado $E_{j}$.

$h_{i j}=$ probabilidad de transitar en un paso cualquiera desde el estado $E_{i}$ al estado $E_{j}$.

La cadena de Markov recién generada está descrita por la siguiente matriz $2 \times 2$, llamada matriz de transición:

$$
\left[\begin{array}{cc}
P_{11}=2 / 3 & p_{12}=1 / 3 \\
P_{21}=0 & p_{22}=1
\end{array}\right]
$$

La solución a la primera cuestión se obtiene mediante la resolución del sistema de ecuaciones $h_{i j}=p_{i j}+\sum_{k \pi j} p_{i k} h_{k j}$, que nos proporciona la siguiente información sobre las probabilidades «tarde o temprano»: $h_{22}=1, h_{12}=1, h_{21}$ $=0$ y $h_{11}=2 / 3$.

El conjunto de probabilidades de que disponemos nos permite clasificar el conjunto de estados en: $E_{2}$ (exterior de la cueva) como estado recurrente y absorbente y $E_{1}$ (interior de la cueva) como estado de transición.

La duración media del proceso aleatorio se obtiene resolviendo el sistema de ecuaciones siguiente: $r_{i j}=1+\sum p_{i k} r_{k j}$, suma que se extiende para los $k \pi j$ y $p_{i k} \pi 0$. Claramente, nos interesa $r_{12}=3$ (duración media del proceso que nos permite alcanzar $E_{2}$ desde $E_{1}$, tarde o temprano).

Los resultados anteriores, obtenidos para un observador, deben ser traducidos en términos del explorador protagonista de la historia del problema. Así: 
1) La probabilidad de salir de la cueva $\left(h_{12}\right)$ es 1 . Así que es seguro que tarde o temprano se sale de la cueva (¿con tal que se viva suficientemente?).

2)El número de exploradores que saldrán de la cueva será 27.

3)El número medio de intentos que han de hacerse para salir de la cueva es 3 .

4) Como las tres puestas pueden ser escogidas con igual probabilidad en cada uno de los intentos, y uno de ellos necesariamente es el que conduce al exterior en 1 hora, es de esperar que los otros dos intentos se produzcan por cada una de las puertas restantes, con una duración respectiva de 2 y 3 días. En consecuencia, el tiempo esperado para que se detenga el proceso (todos los exploradores fuera de la cueva) es de 5 días y 1 hora.

\section{El estrato intermedio del SMS}

El espacio de posibilidades para cada explorador puede ser representado por un árbol infinito, el que se obtiene de continuar los caminos que en el caso finito quedaron cerrados por la restricción de alimentos. De esta manera, excepto para el estado 1 (salir de la cueva en 1 hora), el árbol se construye de la misma manera en los sucesivos intentos, por lo que la probabilidad de salir en el próximo intento es la misma tanto si estamos en el primer intento como en el 200 (propiedad de Markov).

Mientras que los estados 2 y 3 (volver a la cueva después de 2 y 3 días, respectivamente) son distinguibles para cada explorador, para un observador externo del proceso aleatorio, estos estados significan estar dentro de la cueva y, consecuentemente, el estado 1 significa estar fuera de la cueva. Así, pues, para este observador, podemos considerar los estados $E_{1}$ y $E_{2}$ definidos de esta manera:

$E_{1}=$ «Estar dentro de la cueva, no importa en que intento.»

$E_{2}=$ «Estar fuera de la cueva, no importa en que intento se produjo.»

Para estos dos estados, los sucesivos intentos producen las posibilidades que representamos en el siguiente árbol infinito.

Este árbol infinito puede traducirse a un grafo como éste:
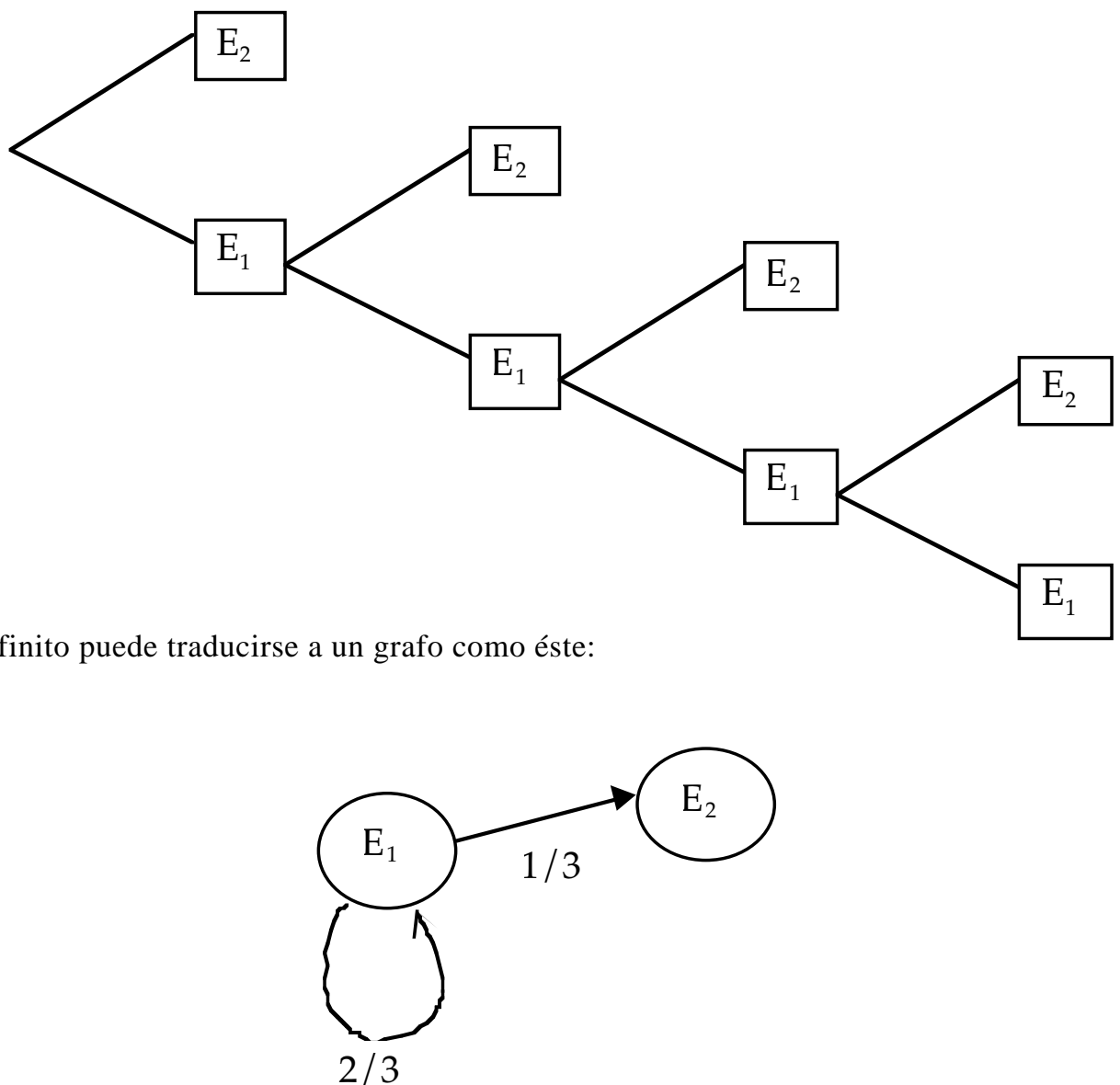
Un grafo como el anterior nos permite clasificar los estados que lo forman. Así, $E_{2}$ es un estado vecino de $E_{1}$, pues puede alcanzarse en un solo paso desde el estado $E_{2}$; del mismo modo, $E_{l}$ es un estado vecino de sí mismo. El estado $E_{1}$ es un estado interior o de transición, pues a él le llegan flechas y también salen de él. El estado $E_{2}$ es, en cambio, recurrente y absorbente, pues a él sólo le llegan flechas y de él no parte ninguna.

Los sistemas de representación que caracterizan este estrato del SMS requieren, como en el caso finito, de reglas de cálculo para responder a las preguntas sobre la probabilidad de alcanzar el estado absorbente $E_{2}$ partiendo desde el estado $E_{1}$ y la duración media del proceso aleatorio. Las reglas conocidas como 1a. y 2a. reglas del valor medio (Engel, 1975b) resuelven estas cuestiones:

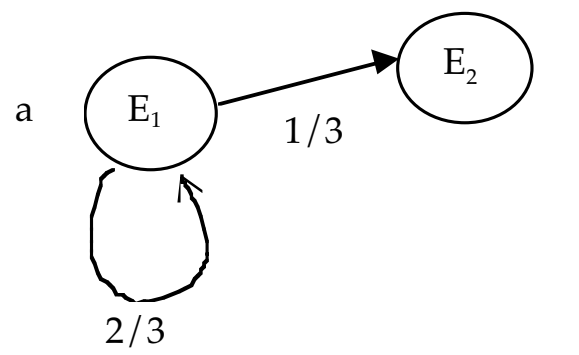

1

Siendo $a=h_{12}$, que la usamos como incógnita auxiliar,y aplicadas las condiciones frontera al estado absorbente $\left(h_{22}=1\right)$, la primera regla del valor medio nos da: $a=2 /$ $3 a+1 / 31$, ecuación de una incógnita cuya solución $a=1$ nos da la probabilidad de alcanzar el estado $E_{2}$ desde el estado $E_{l}$, tarde o temprano.

La duración media del proceso aleatorio exige imponer al grafo las condiciones frontera al estado absorbente $r_{22}=0$. La segunda regla del valor medio aplicada al siguiente grafo nos da la duración media buscada:

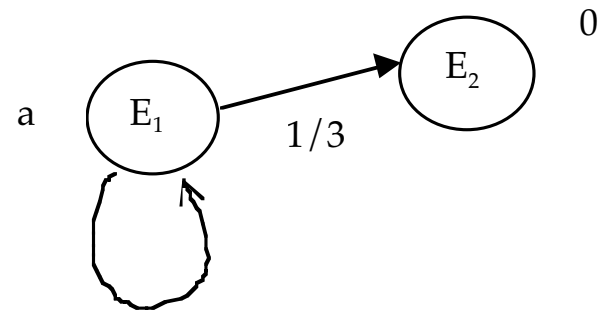

$2 / 3$

$a=r_{12}$

$a=1+2 / 3 a+1 / 3(0)$. De donde $a=3$.

De la misma manera que los datos obtenidos hacen referencia a un observador, procede, como hemos hecho con el anterior estrato del SMS, traducir los resultados para el explorador.

\section{El estrato menos abstracto del SMS}

El espacio de pruebas lo podemos representar mediante uno de los dos grafos siguientes, según sea la referencia del proceso aleatorio, el explorador o un observador externo. Así, para un explorador, el grafo siguiente representa la situación aleatoria que se describe en el enunciado del problema, grafo que incluye además las probabilidades de transición entre estados:

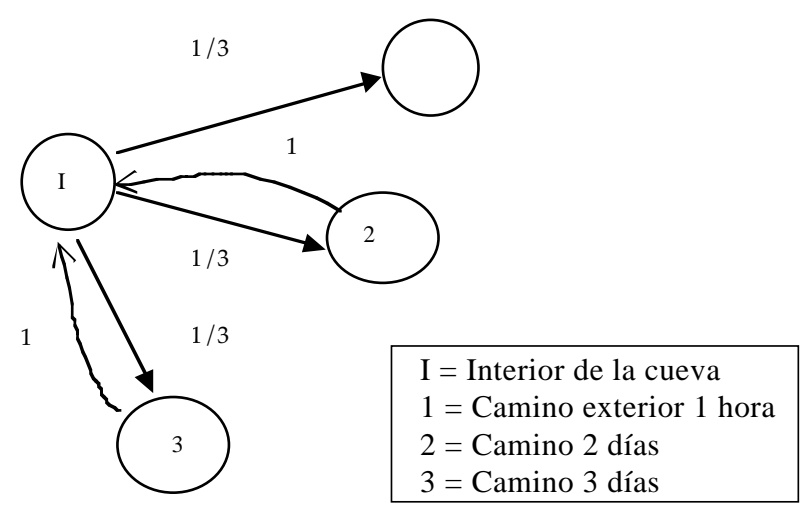

Para un observador externo, el grafo y con las probabilidades es el siguiente:

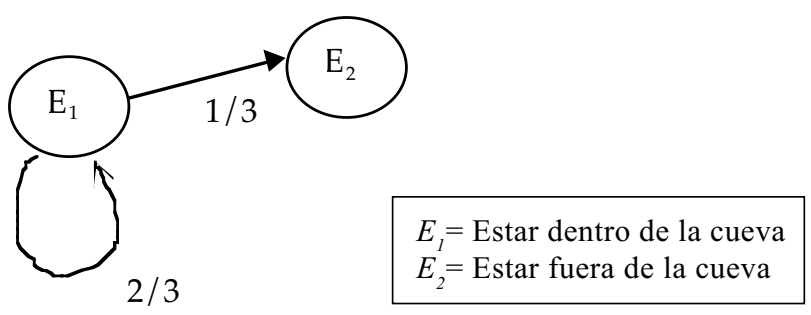

Resolvamos el problema, como hicimos en los casos anteriores, usando la figura del observador y en este caso el grafo que representa el proceso. En el interior situamos los 27 exploradores representados por fichas. Ahora, otro atributo que no sea el número de fichas no es relevante para la resolución del problema. El tablero de juego queda como sigue:

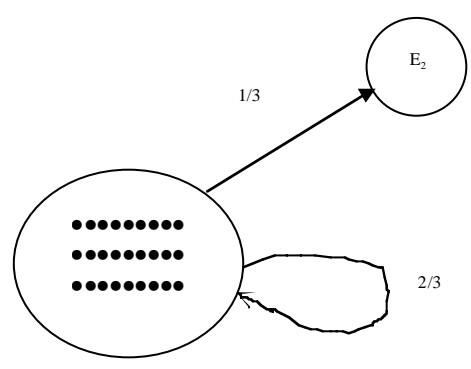

ENSEÑANZA DE LAS CIENCIAS, 2002, 20 (1) 
Las reglas de juego son, inicialmente, importadas desde el caso finito. Así que, después de 75 movimientos ${ }^{6}$, la situación es la que representa el grafo siguiente:

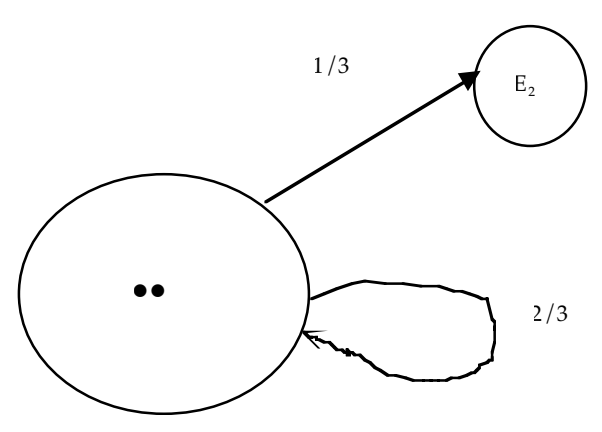

$\bullet \bullet \bullet \bullet \bullet \bullet \bullet$

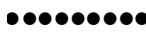

$\bullet \bullet \bullet \bullet \bullet \bullet \bullet \bullet \bullet$

La situación anterior no puede continuarse si solamente se aplican las reglas del juego que hasta ahora se tienen y que responden a una visión frecuentista de la probabilidad. Es necesario romper este impás con el uso del ábaco probabilístico (Engel, 1975b). Así, puede considerarse que el problema es equivalente a un grafo con una carga crítica $^{7}$ de dos fichas, como el que representa la figura anterior. Se introducen fichas en el estado $E_{l}$ y se juega. El proceso o juego acaba cuando, después de mover, el estado cargado críticamente queda como estaba al iniciar el proceso. La serie de grafos siguiente representa el inicio y el final del proceso:
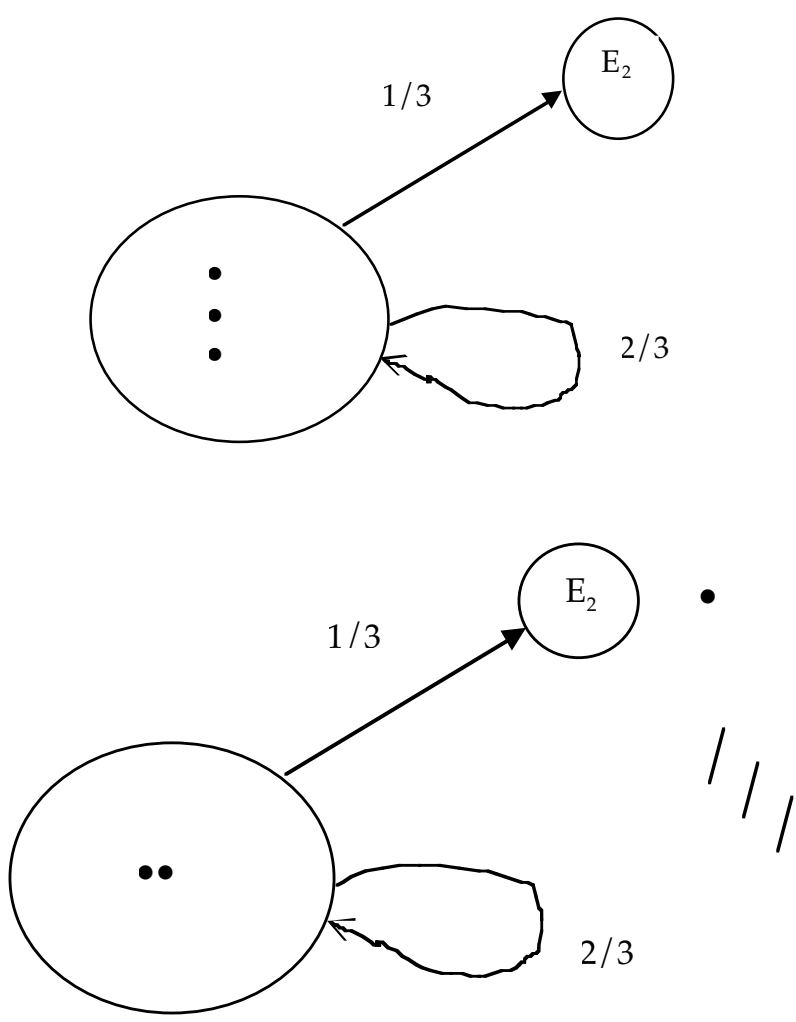

Los resultados que produce la aplicación del ábaco probabilístico deben ser interpretados. Así, se introduce una ficha en el estado $E_{1}$ y es absorbida por el estado $E_{2}$. Para la ficha introducida se realizan tres movimientos y el proceso acaba. Con estos datos, la probabilidad de transitar desde el estado $E_{1}$ al estado $E_{2}$ es 1 , ya que la ficha que se introduce en el estado $E_{l}$ es absorbida por el estado $E_{2}$. Como el número de movimientos es 3, para una ficha absorbida, el número medio de movimientos continuará siendo 3. Estos datos, que son para el observador, deben ser traducidos para el explorador:

- Si la probabilidad de transitar de $E_{1}$ a $E_{2}$ es uno, es seguro que todos los exploradores saldrán de la cueva.

- Si el número medio de movimientos de las fichas es 3 , entonces, por término medio, un explorador realizará tres intentos por salir de la cueva. En consecuencia, igual que hicimos en casos anteriores, es de esperar que estos intentos recorran todos los caminos posibles y el tiempo esperado sea de 5 días y 1 hora.

\section{EL PROBLEMA DE LA CUEVA Y LA SIMU- LACION}

\section{La simulación}

Decía Polya (1966) que todos los problemas que impliquen fenómenos azarosos de masas (de probabilidad) podrían reducirse a problemas con bolsas y bolas, escogidas éstas en cantidades apropiadas. Polya, partiendo de la definición axiomática de la probabilidad de von Mises, considera la naturaleza empírica de la probabilidad de un suceso definida por la frecuencia relativa de las veces que ocurre.

Simular un problema de probabilidad es una destreza con contenido heurístico (DH) (Puig, 1996) que permite resolver el problema de probabilidad por otros medios. Aunque hay una cierta transformación del problema original en el problema simulado, esta transformación no permite considerar el problema transformado como diferente del original, ya que lo que hay que hacer es lo mismo que en el problema original pero usando esta vez una determinada destreza: dados, urnas, números aleatorios. Es por eso por lo que la simulación no debería considerarse como una herramienta heurística $(\mathrm{HH})$ sino como una destreza heurística (DH).

Entre el problema original y el problema simulado debe haber una correspondencia que los haga equivalentes en términos de probabilidad. Así:

1) El espacio de posibilidades del problema original ha de corresponderse en probabilidad con el espacio de posibilidades del problema simulado. Por ejemplo, escoger una de las tres opciones para salir de la cueva tiene probabilidad 1/3. El espacio de posibilidades que puede obtenerse al lanzar un dado cúbico laplaciano considerando los pares $(1,2)$ para la opción $1,(3,4)$ para la 
opción 2 y los pares $(5,6)$ para la opción 3 , permiten establecer la siguiente correspondencia:

El par de resultados $(x, y)$ al lanzar una vez el dado cúbico laplaciano se corresponde con la opción $A$, para $\mathrm{A}=1,2,3$, en el problema de la cueva si $p[(x, y)]=p(A)$. Cada vez que un explorador hace un intento por salir de la cueva, dado que aún no lo ha conseguido, independiente del resultado del intento anterior, se ha de corresponder en los mismos términos de independencia con los lanzamientos consecutivos del dado, si en ningún caso anterior apareció el 1 o el 2.

Esto mismo que hemos hecho con un dado puede hacerse con el generador de azar por excelencia, los números aleatorios, cuyo uso como método de resolución de problemas de probabilidad se conoce con el nombre de método de Monte-Carlo (Engel, 1975a).

2) Cualquier posibilidad en el problema original puede conseguirse en el problema simulado. Así, he de poder salir a la primera, segunda o tercera, en el caso finito, o en cualquier caso, en el caso infinito. Y esto es así porque es posible obtener el 1 o el 2 al primer lanzamiento, al segundo, al tercero, etc.

\section{El proceso de simular, la información y los datos, y el número de simulaciones}

Una simulación consiste en una experimentación del proceso aleatorio que está presente en el problema original mediante el proceso aleatorio que hay en el problema simulado. El proceso de simular consiste, pues, en la obtención de todos los resultados posibles del proceso aleatorio simulado. Veremos después que esto es una dificultad en la tarea.

Lo que se obtiene en cada simulación es información sobre los resultados posibles del proceso aleatorio simulado. Esta información ha de ser traducida en datos para poder dar respuesta al problema simulado. Se requiere, entonces, de un tratamiento estadístico de esos datos -ordenación y representación mediante tablas y gráficos, análisis de los datos mediante medidas de centralización y de dispersión- con el fin de dar respuesta al problema simulado.

Todo tratamiento de fenómenos azarosos de masas, utilizando la terminología de Polya (al menos su traducción), es dependiente del número de simulaciones que se hagan. En consecuencia, la frecuencia relativa asociada a un determinado resultado posible $-\mathrm{y}$, por tanto, la probabilidad así asignada- depende del número $n$ de simulaciones. «¿Cuántas simulaciones debemos de hacer?» es una pregunta muy habitual que hacen los estudiantes. Se responde: «Cuántas más mejor.»Pero esta respuesta, claro está, depende de con qué fines se esté usando la simulación para resolver un problema de probabilidad. Si la uso (profesor) porque no sé qué probabilidad puede tener un determinado resultado, entonces, cuantas más, mejor, ya que (ley fuerte de los grandes números), si pudiera hacer infinita la frecuencia relativa resultante, se podría asociar con su probabilidad en el límite de aquéllas. Es el caso, por ejemplo, de la chincheta. Ahora bien, si sé (profesor) la probabilidad pero los métodos que uso para resolverlos o los fines que persigo aconsejan usar la simulación, entonces, el número de simulaciones que han de hacerse depende de dos cosas (ley débil de los grandes números): del grado de aproximación con el que se quiere que la frecuencia relativa calculada por los estudiantes se aproxime a la probabilidad teórica y del grado de confianza que quiero tener (profesor) de que esto ocurra en mis clases -aunque también de la probabilidad teórica que hay que calcular, pero esto lo dejamos para el final. Así, por ejemplo, si la probabilidad de salir de la cueva es $2 / 3$ y quiero que la aproximación de la $f r_{n}$ a esta probabilidad sea menor que 0,01 con una grado de confianza del $95 \%$, el número $n_{0}$ (número mínimo de simulaciones) a partir del cual esto ocurre se obtiene de $n_{0} \geq 2 / 3 \sum[1-(2 / 3)] / 0,01^{2} \sum 0,05$; es decir, $n_{0} \geq 44.444$ simulaciones. En una clase de 30 alumnos cada uno de ellos debería hacer sin desfallecer $\mathrm{y}$ sin «trampas» un total de 1.481 simulaciones del problema.

El resultado del problema resuelto por simulación, significados asociados por el sentido en el que se usan los conceptos

El primer concepto que vamos a ver es el de probabilidad. La naturaleza de este concepto en un problema simulado es empírica, adquiere el significado de «frecuencia relativa», ya que ésta se usa aquí en este sentido. Por tanto, el alumno (como resolutor del problema) ve en la probabilidad un medio para organizar si un resultado posible es más o menos fácil o difícil de producirse que otro, permaneciendo él al margen de la toma de esta decisión. Los números deciden. Eso hace que sea pertinente preguntar, por ejemplo, si es «fácil salir de la cueva»o «más difícil salir de la cueva que quedarse dentro», en lugar de la «probabilidad de salir de la cueva».

Las medidas de centralización usadas para dar el resultado de un problema por simulación permiten que el concepto de esperanza matemática o de valores esperados adquiera aquel significado, pues aquéllos se están usando en ese sentido. Hay diferencias, no obstante, en el uso de estas medidas de centralización, ya que no es lo mismo usar una media aritmética que una moda o una mediana como esperanza matemática o valor esperado. La media aritmética deja al margen de cualquier decisión al estudiante mientras que el uso de cualquiera de las otras dos puede estar derivado de una decisión previa del estudiante. Así, ante la pregunta «¿Cuántos exploradores esperas que se salven de los 27?», lo razonable en el proceso de resolución del problema de la cueva que usa la simulación es usar la moda con el significado de «valor esperado o esperanza matemática».

\section{De la conveniencia en el uso de la simulación}

El uso de la simulación para resolver problemas de probabilidad depende de muchas cosas. No encuentro 
demasiados argumentos para negar su uso, aunque a veces, para determinados casos, no sea demasiado conveniente.

Imaginemos que, en nuestro problema, queremos calcular la probabilidad de que salgan exactamente 3 exploradores. La probabilidad teórica de este suceso es $3,1 \sum 10^{-9}$. Para obtener esta misma probabilidad empíricamente, el número de simulaciones que habría que hacer, para los parámetros que estamos usando, sería: $\mathrm{n}_{0} \geq 3,1 \sum 10^{-9} \sum\left[1-\left(3,1 \sum 10^{-9}\right)\right] /\left[(0,01)^{-9}\right]^{2} \sum 0,05$. Esto nos da que $n_{0} \geq 6,210^{10}$ simulaciones, es decir, del orden de los diez mil millones de simulaciones. Sin comentarios. Claro que también se puede decir que este suceso es imposible o casi imposible, pero ésta es una razón de otro estilo.

\section{CONCLUSIONES}

Los análisis que hemos expuesto, y otros que se pueden hacer y que más adelante indicaré, pretenden mostrar las maneras de hacer que pueden ser objeto de enseñanza con estudiantes para maestros y profesores de matemáticas. Su ubicación en un temario de formación se correspondería con las tareas que, entre otras, podrían hacerse en didáctica de la probabilidad y la estadística. El problema que hemos usado para ejemplificar los análisis puede usarse, desde el punto de vista metodológico, de más de una manera. Una, como problema en el que desplegar los diferentes análisis que acabamos de hacer, mediante su resolución, y como contexto de enseñanza en el que mostrar de qué manera puede analizarse cada uno de los procesos de resolución, introduciendo, entonces, los elementos de análisis.

En este segundo caso, usando el problema como contexto de enseñanza, los diferentes modelos de competencia que se van construyendo permiten introducir no sólo los elementos para el análisis del proceso de resolución sino otros que también están presentes tanto en el problema como en su resolución. Recordemos algunos de ellos:

- Se discute la naturaleza del azar que está presente en el problema.

- Se analiza la independencia de las pruebas por las que pasa cada explorador en los sucesivos intentos, independencia que puede estudiarse entre los protagonistas del problema: para un explorador y entre exploradores.

- Se toma en consideración la figura del observador del proceso y su papel en la resolución del problema.

- Se consideran diferentes estratos del sistema matemático de signos con los que se expresan tanto los sucesos como sus probabilidades, en función del proceso finito o infinito, y que permiten resolver el problema.

- Se analiza el carácter heurístico de los sistemas de representación que forman parte de esos estratos. Los árboles, por ejemplo, son sistemas de representación que como destreza tienen potencial heurístico, ya que no sólo son útiles para un buen número de problemas, sino que tienen su propia estructura interna y son generadores de nuevos problemas; así mismo, los grafos que representan Cadenas de Markov.

- Se discuten los significados de los diferentes conceptos asociados a la estructura conceptual de la probabilidad que se derivan del uso de los mismos en relación con los diferentes sistemas de signos en el que se expresan.

La distinción entre asignación y cálculo de probabilidades en función de los SMS usados para la resolución del problema conduce, por ejemplo, a un análisis longitudinal entre los estratos del SMS y no sólo dentro de cada uno de ellos. Detallar aquí todos estos análisis daría lugar a otro artículo; no obstante, mostraremos un par de ejemplos:

Usar los estratos más abstractos e intermedios en el proceso de resolución implica necesariamente cálculo de probabilidades, pues con el primero de ellos se ponen en uso dos resultados de la teoría de la probabilidad, la probabilidad de la unión disjunta de sucesos y la probabilidad compuesta o conjunta; y con el intermedio las reglas de la suma y del producto para caminos y árboles. Por el contrario, el uso del estrato menos abstracto permite al resolutor asignar probabilidades una vez terminado el proceso. Si consideramos que aprender matemáticas es también ser competente con los diferentes estratos de los SMS, una enseñanza coherente con esto último potenciaría el aprendizaje en el sentido inverso al que hemos mostrado en la exposición, es decir, comenzando con el SMS menos abstracto y favoreciendo el uso de los demás estratos en un orden creciente de abstracción.

El segundo ejemplo lo referiremos a una propiedad, la propiedad de Markov. Formalmente, esta propiedad está expresada dentro del SMS más abstracto como una probabilidad condicional que implica a estados de un sistema que está sujeto a un determinado proceso aleatorio. Es necesario reconocer previamente esta propiedad para poder resolver el proceso aleatorio infinito como una cadena de Markov. Con el estrato intermedio, la propiedad de Markov se reconoce por cómo se construye el árbol infinito o el grafo, al que puede traducirse el árbol infinito, que da cuenta del proceso aleatorio. Siendo el grafo de la manera que es, se pueden aplicar las reglas de cálculo que permiten resolver un grafo como tal que representa una cadena de Markov. Finalmente, con el estrato menos abstracto, la propiedad de Markov es todavía más fácilmente reconocible por el hecho de que la ficha está en un estado o en otro independientemente de la historia previa del proceso, de tal manera que una ficha en el estado dentro de la cueva está en la mismas condiciones, en términos de probabilidad, de salir de la cueva que cuando se inició el proceso o después de un buen número de pruebas y que le mantienen en este estado.

El trabajo que aquí se ha descrito abre, en nuestra opinión, muchas posibilidades de continuidad, ahora 
investigando, por ejemplo, a los resolutores en los diferentes niveles educativos con el fin de analizar niveles de competencia en el uso de los estratos del SMS, éstos que se han mostrado aquí y otros que podrían derivarse de esos estudios.

\section{NOTAS}

${ }^{1}$ Aunque se podría, no me parece razonable hacer una distinción explícita de si la formación se refiere a maestros de enseñanza primaria o profesores de matemáticas de enseñanza secundaria. Aquí me referiré a formación de profesores de matemáticas, entendiendo por ello que se habla de formación de estudiantes para maestros o profesores que tendrán, entre sus tareas, la tarea de enseñar matemáticas. Solamente el uso que se pueda hacer de los análisis que se van a realizar en este artículo determinarán el nivel educativo de los estudiantes, tanto de profesor de primaria como de secundaria.

${ }^{2}$ El estudiante de profesor de matemáticas ha de resolver los problemas que sus estudiantes resolverán con posterioridad. Durante su formación, lo que mis estudiantes resuelven son lo que llamo problemas con intención didáctica, como el que nos

\section{REFERENCIAS BIBLIOGRÁFICAS}

ENGEL, A. (1975a). L'enseignement des probabilités et de la statistiques, (2 vols.). Cedic: París. Trad. de Engel, A. 1988. Probabilidad y estadística (2 vols.). Mestral: Valencia.

ENGEL, A. (1975b). The probabilistic abacus. Educational Studies in Mathematics, 6, pp. 1-22.

FILLOY, E. (1999). Aspectos teóricos del álgebra educativa. México: Grupo Editorial Iberoamericana.

FISCHBEIN, E. (1977). Image and Concept in Learning Mathematics. Educational Studies in Mathematics, 8(2) pp. 153-165.

GORDON,H.(1997). Discrete Probability. Nueva York: Springer Velag.

GRUPOCERO(1995). Matemáticas para la secundaria obligatoria. Madrid: MEC-Edelvives. ocupa, cuyo enunciado incluye el problema original y aborda, además, cuestiones didácticas que tienen que ver con el propio contenido matemático implicado, -que puede ser objeto de aprendizaje- y con los procesos de resolución de problema, que también puede serlo.

${ }^{3}$ Un componente nulo indica el fin del proceso aleatorio con dos posibilidades, dentro o fuera de la cueva.

${ }^{4}$ Usamos el término árbol de longitud 3 para indicar que las ramas del árbol se extienden como máximo hasta la tercera posibilidad.

${ }^{5}$ La figura del observador externo del proceso aleatorio, distinta de la figura del protagonista de la historia del problema, la usaremos más de una vez a lo largo de los procesos de resolución del problema, ya que su consideración nos permitirá introducir sistemas de representación que simplifican el proceso.

${ }^{6}$ Un movimiento de una ficha representa el resultado de un intento de un explorador por salir de la cueva.

${ }^{7}$ Un estado se dice que está cargado críticamente si el número de fichas es una menos que las necesarias para poder mover (Engel, 1975b). Como las probabilidades de transición entre estados son $1 / 3$ y $2 / 3$, la carga crítica será de dos fichas.

HUERTA, M.P.(2000). Didàctica de la probabilitatil'estadística. Servei de publicacions de la Universitat de València: Valencia, documento presentado para su publicación.

POLYA, G. (1966). Matemáticas y razonamiento plausible. Madrid: Tecnos.

PUIG, L. (1996). Elementos de resolución de problemas. Granada: Mathema.

PUIG, L. (1997). Análisis fenomenológico, en Rico, L. (coord.). La educación matemática en la enseñanza secundaria. Cuadernos de formación del profesorado, 12. ICE Universitat de Barcelona, pp. 61-94. Barcelona: Horsori.

PUIG, L. y CERDÁN, F. (1988). Problemas aritméticos escolares. Madrid: Síntesis.

SHAUGHNESSY, J.M. (1992). Research in Probability and Statistics: Reflections and Directions, en Grouws, D. (ed.). Handbook of Research on Mathematics Teaching and Learning, pp. 465-494. Nueva York: Macmillan Publishing Company.

[Artículo recibido en diciembre de 2000 y aceptado en junio de 2001.] 